\title{
A NOVEL PRIVACY PRESERVATION MECHANISM FOR DATA AND USER IN DISTRIBUTED SERVERS
}

\author{
Dr.D.Suresh ${ }^{1}$, Dr.N.Dhanalakshmi ${ }^{2}$, Dr.A.Thomas Paul Roy ${ }^{3}$ \\ ${ }^{1}$ Professor, PSNA College of Engineering and Technology, Dindigul, India \\ ${ }^{2}$ Professor, PSNA College of Engineering and Technology, Dindigul, India \\ ${ }^{3}$ Professor, PSNA College of Engineering and Technology, Dindigul, India \\ suresh@psnacet.edu.in
}

\begin{abstract}
Advances in sensing and monitoring science allow location-based purposes however they additionally create tremendous privateness risks. Anonymity can supply a excessive diploma of privacy, retailer provider customers from dealing with carrier providers' privateness policies, and limit the carrier providers' necessities for safeguarding non-public information. However, guaranteeing nameless utilization of location-based offerings requires that the particular region facts transmitted via a person can't be without difficulty used to re-identify the subject. This paper provides a middleware structure and algorithms that can be used by using a centralized place dealer service. The adaptive algorithms regulate the decision of region data alongside spatial or temporal dimensions to meet distinct anonymity constraints based totally on the entities who can also be the use of place offerings inside a given area. Using a mannequin based totally on car site visitors counts and cartographic material, we estimate the realistically anticipated spatial decision for extraordinary anonymity constraints. The median decision generated with the aid of our algorithms is a hundred twenty five meters. Thus, nameless location-based requests for city areas would have the identical accuracy presently wanted for E-911 services; this would supply enough decision for wayfinding, automatic bus routing offerings and comparable locationdependent services.
\end{abstract}

\section{INTRODUCTION}

Cloud computing is an increasing computing paradigm that attracts growing interest in each search and business community. The externalization of records and calculations to cloud servers provides a cost-beneficial way of conducting mass storage and processing of questions. However, because of privacy concerns, touching facts are as correct as other unauthorized users to be covered from the cloud server. The encryption of the data is a common way of protecting the confidentiality of outsourced information. In addition, licensed clients send encrypted queries to the cloud server to protect the confidentiality of the question. It shows our distressed situation of closely closed question processing over encrypted cloud facts. The proprietor of statistics outsources the cloud server with encryption information. Cloud server technology encrypts customer queries about the encrypted facts and returns the result of questions to the customer. The cloud server must not now gain expertise in the data, data patterns, query results or question results during question processing. Fully homomorphic encryption schemes guarantee robust security while enabling arbitrary data calculations. The calculation fee, however, is practically prohibitive. Trusted hardware such as SGX provides a successful alternative to Intel's Software Guard Extensions, but still has barriers in their security guarantees. Many strategies were proposed to support unique queries or calculations of encrypted facts with different levels of safeguards and effectiveness (e.g., by using weaker encryptions). A 
substantial study was conducted with search for similarities, invulnerable k-nearest neighbor(KNN) queries that return the most (closest) archive of a record of questions.

\section{II.LITERATURESURVEY}

Advances in sensing and monitoring technological know-how allow location-based purposes however they additionally create sizable privateness risks. Anonymity can supply a excessive diploma of privacy, shop provider customers from dealing with provider providers' privateness policies, and minimize the provider providers' necessities for safeguarding personal information. However, guaranteeing nameless utilization of location-based offerings requires that the particular vicinity facts transmitted by means of a consumer can't be without problems used to re-identify the subject.

This paper gives a middleware structure and algorithms that can be used through a centralized place broking service. The adaptive algorithms regulate the decision of vicinity statistics alongside spatial or temporal dimensions to meet unique anonymity constraints based totally on the entities who might also be the use of vicinity offerings inside a given area. Using a mannequin based totally on car visitors counts and cartographic material, we estimate the realistically anticipated spatial decision for distinctive anonymity constraints. The median decision generated through our algorithms is one hundred twenty five meters. Thus, nameless location-based requests for city areas would have the identical accuracy presently wanted for E-911 services; this would supply enough decision for wayfinding, computerized bus routing offerings and comparable location-dependent services[1].

The fact that dummies can always be available is useful for improving the success price, but using dummies is a major problem. First, how to create a stupidity which is unlike a real consumer, especially on road networks with various movements. Secondly, dummies can be utilized for launching attacks on a completely server-based area by using malicious buyers that influence the providers' company. In this article, we advise a new consumer-oriented system to keep the community carrier in check always, which also succeeds in protecting location mainly based servers against attacks[2]. We used an offline trajectory grouping algorithm that divided the path of users into a predictable, reusable practical images on avenue network using derived parameters. We have developed a privacy protocol to control the actions of all consumers privately in order to overcome malicious consumer by using dummies to launch assaults on areas based on totally servers. We have investigated the effectiveness of our algorithm with some comparative metrics described and provided superb protection of privacy, comfortable purchasers in all circumstances at a reasonable dumb fee when constantly querying road community service.

The growing use of cell gadgets has induced the improvement of vicinity based totally offerings (LBS[3]. By presenting vicinity data to LBS, cellular customers can revel in range of beneficial purposes utilising place information, however would possibly go through the troubles of personal statistics leakage. Location records of cell customers wishes to be saved secret whilst preserving utility to acquire appropriate provider quality. Existing place privacy improving methods based totally on $K$-anonymity and Hilbertcurve cloaking vicinity era confirmed blessings in privateness safety and provider first-class however risks due to the technology of giant cloaking areas that makes question processing and verbal 
exchange much less effective. In this paper we suggest a novel region privateness maintaining scheme that leverages some differential privateness primarily based notions and mechanisms to submit the most useful dimension cloaking areas from more than one circled and shifted variations of Hilbert curve. With experimental results, we exhibit that our scheme extensively reduces the common dimension of cloaking areas in contrast to preceding Hilbert curve method. We additionally exhibit how to quantify adversary's capacity to function an inference assault on consumer region information and how to restriction adversary's success fee below a designed threshold.

Location-Based Service (LBS) has end up a necessary phase of our every day life. While playing the comfort furnished via LBS, customers might also lose privateness considering the untrusted LBS server has all the facts about customers in LBS and it may also tune them in a number approaches or launch their private information to 0.33 parties. To tackle the privateness issue, we suggest a DummyLocation Selection (DLS) algorithm to reap k-anonymity for customers in LBS. Different from present approaches, the DLS algorithm cautiously selects dummy places thinking about that aspect statistics may additionally be exploited with the aid of adversaries. We first pick these dummy areas based totally on the entropy metric, and then advocate an enhanced-DLS algorithm, to make certain that the chosen dummy places are unfold as some distance as possible. Evaluation effects exhibit that the proposed DLS algorithm can extensively enhance the privateness degree in phrases of entropy. The enhanced-DLS algorithm can make bigger the cloaking place whilst preserving comparable privateness degree as the DLS algorithm[4-6].

\section{III.PROPOSED SYSTEM}

\section{A Skyline}

A skyline query returns objects that cannot be controlled by various objects. In the case of a multidimensional objects dataset, an item dominates an object in at least one dimension if it is as appropriate in all dimensions.

Skyline queries obtained wonderful interest in the database neighborhood all through the previous decades. The skyline computation grew to be quintessential to many multi-criteria choice making applications. A good sized variety of algorithms have been proposed and studied extensively.

\section{B Stegnography Data}

Steganography is a hidden statistical artwork in a seemingly innocuous medium of cowl. For instance, a digital image can hide any tactile statistics internally. Because of the encryption, the contents of the message are now not contained in the message, steganography offers more security than encryption.

Steganography is a work of hiding facts and an attempt to disguise information that is embedded. It serves to secure messages better than cryptography which only hides the content of the message, now that the message does not exist. The original message is hidden inside a service so that no adjustments occur in the service. We will discuss in this paper how digital pixs are used to hide messages. In addition, this paper analyzes the overall performance of certain steganographic instruments. Steganography is a useful tool for covered statistical transmission via the communications channel. The hidden image is provided by combining secret photos 
with the photo provider. The hidden photograph is difficult to understand, apart from recovery. This paper will be detailed by introducing the reader with more than a few ideas of Steganography, a brief record of Steganography and a part of the Steganography technology.

\section{Blockchain Privacy}

A blockchain is a list of records that are linked to the use of cryptography on the first block chain. A cryptographical hash, a time stamp and the transaction information are contained within each block (generally represented as a Merkle tree).

A blockchain is resistant to data modification by design. It is "an open and assigned directory that can properly and verifiably and forever file transactions between two events." A blockchain is commonly used as a disbursed ledger with the aid of an internode verbal exchange protocol and validation of new blocks, which is used at the same time as an inter-node. When recorded, the information in any block cannot be retroactively altered, so that the subsequent blocks cannot be altered, which requires a majority agreement. Although blockchain archives are not unchanged anymore, block chains can be viewed imperfectly through a sketch, and show an excessively byzantine fault-tolerance dispensed computer gadget. Therefore, a blockchain claimed decentralized consensus.

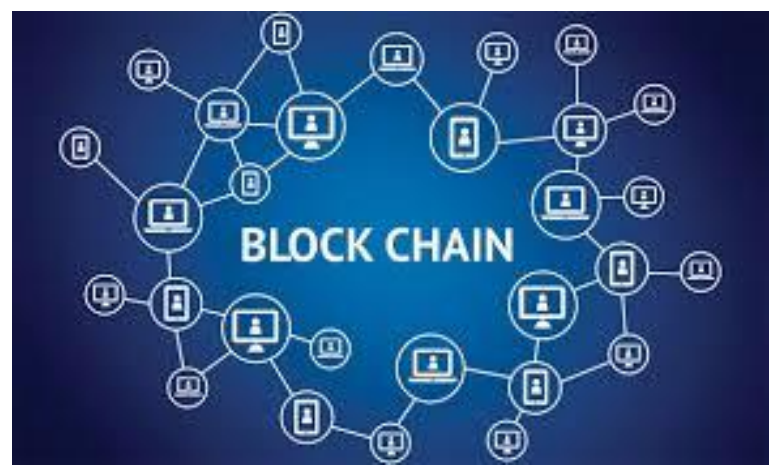

\section{SecureMulti-party Computation (SMC)}

Secure multi-party computing is a subfield of cryptography (also considered as impenetrable computing, mixed computer (MPC) or computing which preserves privacy) in order to develop strategies for events which can calculate each other features through their inputs while preserving these inputs in private. Contrary to the usual cryptographic tasks, cryptography ensures protection and integrity of verbal exchange or storage, and the adversary is the devices of the members (an eavesdropper on the sender and the receiver) outside.

A work on intellectual poker, cryptographic work simulating sport play/computational duties over distances that require a dependent party of 0.33 , begun the basis for unassailable multiparty computation in the late seventies. Note that cryptography has traditionally been about concealing content, while this new type of calculation and protocol involves concealing partial statistics on statistical data while calculating data from many sources and producing output efficiently. 


\section{E Secure Query Processing On Encrypted Data}

Fully homomorphic encryption schemes allow arbitrary computations on encrypted data. Even even though it is proven that we construct encryption schemes, to furnish higher protection the information has to be encrypted. Many methods are proposed to help computations on encrypted statistics with safety warranty and efficiency. We are conscious of intruder in any formal work on invulnerable skyline queries over encrypted information with semantic security.

An essential lookup has been made to reply the trouble that customers might also be fascinated for skyline queries in subspaces of the data. In a framework is proposed which makes use of skyline organizations and decisive subspaces, to compute the skyline in any required subspace. Upon this framework an environment friendly algorithm is proposed, named SKYEY, which applies a top-down method to recursively compute the skyline in subspaces. Pre-sorting techniques and multidimensional roll-up and drill-down evaluation limit the set of objects to be searched. A comparable approach, the SKYCUBE, is proposed in, which is the union of the skylines of all feasible non-empty subsets of a given set of dimensions. Several computation sharing techniques are used, primarily based on correctly figuring out the computation dependencies amongst a couple of associated skyline queries. Bottom-Up and Top-Down algorithms are proposed to compute the SKYCUBE efficiently

\section{IV . RESULT}

As for future work, we design to optimize the conversation time complexity to in addition enhance the overall performance of the protocol. Additional facets like encryption and decryption the usage of cryptography as properly as pictures and movies can additionally be carried out in case of phantasm data. Stegnography methods can additionally be applied for higher future enhancement.

\section{CONCLUSION}

In this paper, we proposed a entirely impervious skyline protocol on encrypted records the use of two non-colluding cloud servers beneath the semi-honest model. It ensures semantic protection in that the cloud servers knows nothing about the information together with oblique records patterns, query, as nicely as the question result. In addition, the patron and records proprietor do now not want to take part in the computation. We additionally introduced a invulnerable dominance protocol which can be used through skyline queries as properly as different queries. Furthermore, we proven two optimizations, information partitioning and lazy merging, to in addition limit the computation load. Finally, we introduced our implementation of the protocol and established the feasibility and effectively of the solution. Along with this we introduce extra new techniques like intruder breach, phantasm records occurrences and the encrypted facts as nicely as the encrypted records and database. So that the facts that has been saved in the server are with quiet higher privateness and security. 


\section{VI.REFERENCES}

[1].K. Fawaz and K. G. Shin, "Location privacy protection for smartphone users," in Proceedings of the ACM SIGSAC Conference on Computer and Communications Security, 2014, pp. 239-250.

[2].K. Fawaz, H. Feng, and K. G. Shin, "Anatomization and protection of mobile apps' location privacy threats," in fUSENIXg Security Symposium, 2015, pp. $753-768$.

[3].B. Niu, Q. Li, X. Zhu, G. Cao, and H. Li, “Achieving k-anonymity in privacyaware location-based services," in IEEE INFOCOM, 2014, pp. 754-762.

[4].P.-R. Lei, W.-C. Peng, I.-J. Su, C.-P. Chang et al., "Dummy-based schemes for protecting movement trajectories," Journal of Information Science and Engineering, vol. 28, no. 2, pp. 335-350, 2012.

[5].T. Hara, A. Suzuki, M. Iwata, Y. Arase, and X. Xie, "Dummy-based user location anonymization under real-world constraints," IEEE Access, vol. 4, pp. 673-687, 2016.

[6].T. Wang, J. Zeng, M. Z. A. Bhuiyan, H. Tian, Y. Cai, Y. Chen, and B. Zhong, "Trajectory privacy preservation based on a fog structure for cloud location services," IEEE Access, vol. 5, pp. 7692-7701, 2017. 